\title{
Capacidad funcional, densidad mineral ósea y marcadores de neoformación-reabsorción ósea en pacientes menores de 18 años con movilidad reducida
}

\section{Functional capacity, bone mineral density and neoformation-resorption bone markers in patients under 18 years of age with reduced mobility}

\author{
Enrique Palomo Atance ${ }^{a}$, Elena Medina Cano ${ }^{b}, M^{a}$ Luisa León Sánchez ${ }^{b}$, José Ramón Muñoz-Rodríguez \\ Ana Rodríguez González ${ }^{\mathrm{b}}$, Celia Montoliú Peco ${ }^{\mathrm{b}}$
}

aUnidad de Endocrinología Pediátrica. Hospital General Universitario de Ciudad Real, España
bServicio de Rehabilitación. Hospital General Universitario de Ciudad Real, España
cUnidad de Investigación Translacional. Hospital General Universitario de Ciudad Real, España

Recibido: 27 de mayo de 2019; Aceptado: 18 de noviembre de 2019

¿Qué se sabe del tema que trata este estudio?

La movilidad reducida asociada a distintas enfermedades neuromusculares produce desde los primeros años de vida un aumento de la reabsorción ósea lo que conlleva una disminución de la densidad mineral ósea y un aumento del riesgo de fracturas.
¿Qué aporta este estudio a lo ya conocido?

Este estudio aporta informacion en pacientes pediátricos con diferentes enfermedades neuromusculares, evaluando la relación entre densidad mineral ósea, parámetros bioquímicos de neoformación y reabsorción ósea, y la disminución de la capacidad motora cuantificada mediante una escala funcional validada.

\section{Resumen}

Introducción: La inmovilización prolongada asociada a diversas enfermedades neurológicas, causa osteoporosis secundaria con fracturas patológicas y dolor óseo persistente Objetivos: Establecer la asociación entre densidad mineral ósea (DMO), marcadores de neoformación y reabsorción ósea y grado de capacidad funcional en pacientes menores de 18 años con movilidad reducida. Pacientes y Método: Estudio transversal, realizado entre 1/1/2016 y 31/12/2017 en pacientes de 6 a 18 años diagnosticados de distintas enfermedades neurológicas en Ciudad Real (España). Se analizaron las variables biodemográficas, capacidad funcional según la Functional Mobility Scale (FMS), que valora la movilidad en 5,50 y 500 metros, DMO, 25-hidroxi-vitamina D, fosfatasa alcalina, osteocalcina en sangre y telopéptido amino terminal de cadena cruzada de colágeno tipo I en orina (NTX-I). Se expresan DMO, fosfatasa alcalina, osteocalcina y NTX-I en Z score según valores de referencia para
Palabras clave:

Densidad mineral ósea; vitamina $D$; densitometría; movilidad reducida; reabsorción ósea 
edad y sexo. Se utilizaron estadísticas descriptivas y correlaciones de Pearson y Spearman. Resultados: 36 pacientes (52,7\% niñas), edad media de 8,6 \pm 4,7 años. Valor medio de FMS: 5,3 sobre 18. DMO media: $-1,99 \pm 1,7$ desviaciones estándar (DE), fosfatasa alcalina media: $-2,64 \pm 1,08$, osteocalcina media: $-2,15 \pm 1,39$, y NTX-I medio: $+3 \pm 1,72$. Hubo asociación significativa entre DMO y FMS para 5 metros $(r=0,395 ; p=0,017)$ y para la puntuación total $(r=0,365 ; p=0,029)$. No se encontraron diferencias significativas según estadios de desarrollo puberal. Conclusiones: En la población estudiada se observa disminución en la DMO y en marcadores de neoformación ósea y elevación de marcadores de reabsorción ósea sin asociación con el desarrollo puberal. Los pacientes con menor grado de movilidad presentan una DMO inferior.

\section{Abstract}

Introduction: Prolonged immobilization associated with several neurological disorders causes secondary osteoporosis with pathological fractures and persistent bone pain. Objectives: To establish the association between bone mineral density (BMD), neoformation and bone resorption markers and the degree of functional capacity in children under 18 years of age with reduced mobility. Patients and Method: Cross-sectional study conducted in Ciudad Real, Spain between January 1, 2016, and December 31, 2017 with patients aged between 6 and 18 years diagnosed with different neurological disorders. The following variables were analyzed: age, sex, pubertal stage, functional capacity according to the Functional Mobility Scale (FMS), which assesses the ability to walk from 5, 50 to 500 meters, BMD, 25-hydroxy-vitamin D, alkaline phosphatase and osteocalcin in blood, and N-terminal telopeptide crosslinks in collagen type I (NTX-I) in urine. BMD, alkaline phosphatase, osteocalcin, and NTX-I values are expressed in Z score according to reference values for age and sex. The Pearson and Spearman correlations were used for data analysis. Results: 36 patients $(52.7 \%$ girls $)$ with an average age of $8.6 \pm 4.7$ years. Mean FMS value: 5.3 out of 18 . Mean BMD: $-1.99 \pm 1.7$ standard deviations (SD), mean alkaline phosphatase: $-2.64 \pm 1.08$, mean osteocalcin: $-2.15 \pm 1.39$, and mean NTX-I: $+3 \pm 1.72$. There was a significant association between BMD and FMS for 5 meters $(r=0.395$; $\mathrm{p}=0.017)$ and for total score $(\mathrm{r}=0.365 ; \mathrm{p}=0.029)$. There were no significant differences according to the stages of pubertal development. Conclusions: In this population, there was a decrease in BMD and bone neoformation markers, and an increase of bone resorption markers with no association with pubertal development. Patients with a lower degree of mobility present a lower BMD.

\section{Keywords:}

Bone mineral density; vitamin $\mathrm{D}$; densitometry; mobility limitation; bone resorption

\section{Introducción}

Las fuerzas mecánicas aplicadas al hueso a través del movimiento resultan esenciales para conseguir una adecuada mineralización ósea ${ }^{1}$. Por esta razón la inmovilización prolongada asociada a diversas enfermedades neurológicas es una causa de osteoporosis secundaria con fracturas patológicas y dolor óseo persistente ${ }^{2}$, lo que a su vez puede empeorar la situación funcional de estos pacientes. Se ha observado que la fuerza muscular ejercida durante el movimiento produce una remodelación ósea como respuesta que condiciona la forma y la geometría de los huesos ${ }^{3}$, de modo que la inactividad física va a afectar a esta unidad funcional hueso-músculo produciendo una disminución en la aposición perióstica, sobre todo a nivel de huesos largos como el fémur y la tibia ${ }^{4}$. La explicación fisiopatológica a este hecho estaría en que se alteraría el equilibrio entre neoformación y reabsorción ósea en favor de ésta última, de forma que la inmovilización produciría un aumento de la actividad osteoclástica mediada a través del receptor activador nuclear kappa
B (RANKL) $)^{5}$. Todo ello daría lugar a la presencia de huesos con un menor espesor cortical, y por tanto con un mayor riesgo de fractura ${ }^{6}$.

Por su parte, el aumento de la esperanza de vida en los pacientes diagnosticados de enfermedades neuromusculares a lo largo de los últimos años hace que los problemas relacionados con la salud ósea en estos casos sean cada vez más prevalentes ${ }^{7}$.

El hecho de que la adquisición de la masa ósea tenga lugar durante las dos primeras décadas de la vida ${ }^{8}$ hace que el estudio en este periodo sea determinante para desarrollar estrategias diagnósticas y terapéuticas que eviten consecuencias de mayor gravedad en años posteriores.

Aunque es conocida la relación entre la movilidad reducida y la aparición de alteraciones clínicas y bioquímicas relacionadas con el aumento de la reabsorción ósea, hasta el momento no se ha estudiado en pacientes pediátricos con diferentes enfermedades neuromusculares la relación entre densidad mineral ósea, parámetros de neoformación y reabsorción ósea, y la disminución de la capacidad motora cuantificada 
mediante una escala funcional validada internacionalmente. Por todo ello, el presente trabajo tiene como objetivo principal establecer la asociación entre densidad mineral ósea, marcadores de neoformación y reabsorción ósea y grado de capacidad funcional en un grupo de pacientes menores de 18 años con movilidad reducida. A su vez, como objetivos secundarios, se establecen: conocer si existen diferencias en la densidad mineral ósea y en los marcadores de neoformación y reabsorción ósea en función del desarrollo puberal, así como estudiar la relación entre la densidad mineral ósea y el índice de masa corporal (IMC) en nuestra muestra.

\section{Pacientes y Método}

Estudio transversal. La recolección de datos se efectuó desde el 1 de enero de 2016 al 31 de diciembre de 2017. Para su participación en el estudio se consideraron aquellos pacientes del área sanitaria perteneciente al Hospital General Universitario de Ciudad Real (España) y que estaban en seguimiento por las Unidades de Endocrinología pediátrica y de Medicina Física y Rehabilitación dentro del periodo establecido. Se establecieron como criterios de inclusión: edad de entre 6 y 18 años y estar diagnosticado de distintas enfermedades neurológicas con movilidad reducida asociada según la puntuación del Functional Mobility Scale $(\mathrm{FMS})^{9}$.

Se excluyeron los pacientes que habían recibido tratamiento de mantenimiento con corticoides, anticonvulsivantes, suplementos de calcio o vitamina D y bifosfonatos. Una vez considerados todos estos criterios de inclusión y exclusión, y ante la posibilidad de disponer únicamente de un número limitado de pacientes que imposibilitara la obtención de una muestra ideal, los participantes en el estudio fueron seleccionados mediante un muestreo consecutivo no probabilístico.

Las variables analizadas fueron: edad cronológica, sexo, peso, talla, IMC, desarrollo puberal según los estadios de Tanner, edad ósea según el atlas de Greulich \& Pyle, densidad mineral ósea determinada por absorciometría con rayos X de doble energía (DXA) a nivel de columna lumbar (L2-L4), capacidad funcional según la FMS, valores en sangre de 25-hidroxi-vitamina $\mathrm{D} 3$, paratohormona $(\mathrm{PTH})$, fosfatasa alcalina total y osteocalcina y valores en orina del telopéptido aminoterminal del colágeno tipo I (NTX-I). Se establecieron como parámetros de neoformación ósea la fosfatasa alcalina total y la osteocalcina y como parámetro de reabsorción ósea el NTX-I.

Las variables edad, sexo, peso, talla, IMC y desarrollo puberal se obtuvieron directamente por un mismo investigador mediante la anamnesis y la exploración física de los pacientes, obteniéndose el peso y la talla desde una misma báscula y un mismo tallímetro. Los resultados del IMC se expresaron en $Z$ score según valores de referencia para población pediátrica española ${ }^{10}$.

Functional Mobility Scale (FMS) ${ }^{9}$ es una escala validada para pacientes de 6 a 18 años con enfermedad neuromuscular, que valora la capacidad para desplazarse en tres ámbitos de la vida cotidiana: el domiciliario (desplazamientos en distancias de hasta 5 metros), el escolar (desplazamientos en distancias de hasta 50 metros) y el comunitario (desplazamientos en distancias de hasta 500 metros).

Los datos de la escala del FMS se obtuvieron mediante observación directa del investigador para la distancia de hasta 5 metros (ámbito domiciliario) y mediante entrevista al paciente o cuidador para las distancias de hasta 50 y 500 metros (ámbitos escolar $y$ comunitario respectivamente). Para cada distancia se asignaron 6 puntos si el paciente se desplazaba sin ninguna limitación, 5 puntos si podía caminar sin ayuda pero no podía correr, 4 puntos si precisaba bastones de mano para los desplazamientos, 3 puntos si precisaba bastones ingleses, 2 puntos si precisaba andador, 1 punto si podía desplazarse con silla de ruedas y 0 puntos si no podía desplazarse de forma autónoma en ningún caso. La determinación de la densidad mineral ósea se realizó mediante el sistema Hologic ${ }^{\circledR}$ expresándose los resultados en $\mathrm{Z}$ score para sexo y edad ósea según los valores de referencia de nuestra población ${ }^{11}$ tras aplicar el factor de corrección correspondiente. Los valores de fosfatasa alcalina total, osteocalcina y NTX-I se expresaron en Z score para edad, sexo y estadio puberal según los valores de referencia en edad pediátrica ${ }^{12-14}$. Respecto a la vitamina $\mathrm{D}$, se definió déficit si niveles de 25-hidroxi-vitamina D3 $<12 \mathrm{ng} / \mathrm{ml} \mathrm{e}$ insuficiencia si niveles de 25-hidroxi-vitamina D3 $\geq 12$ $\mathrm{ng} / \mathrm{ml} \mathrm{y}^{2}<20 \mathrm{ng} / \mathrm{ml}^{15}$. Todas las variables analíticas se obtuvieron a primera hora de la mañana con los pacientes en ayunas siguiendo los protocolos de recogida y procesado de muestras del laboratorio de Análisis Clínicos del Hospital General Universitario de Ciudad Real. Para la determinación de la fosfatasa alcalina total se empleó la espectrofotometría cinética (método de Bessey-Lowry optimizado), para la medición de la osteocalcina se empleó el kit "N-Med Osteocalcin" por enzimoinmunoanálisis (ELISA), para la cuantificación del NTX-I el kit beta-Crosslaps Elecsys y, por último, para la determinación de la 25-hidroxi-vitamina D3 la quimioluminiscencia (Immulite 200).

\section{Análisis estadístico}

El análisis de los datos se realizó con el software SPSS v.19.0. Los resultados del estudio estadístico descriptivo se definieron mediante estadísticos de frecuencia central y de dispersión, representándose mediante porcentajes y gráficas de histogramas. 
Respecto al análisis estadístico inferencial, se comprobó la bondad de ajuste con la distribución normal de las variables cuantitativas mediante la prueba de Shapiro-Wilk. Para establecer las correlaciones entre variables, en los casos en los que se obtuvo un buen ajuste con la normalidad se aplicó la correlación de Pearson, y en el resto de casos se recurrió a la correlación de Spearman. Los datos se estratificaron según los diferentes estadios de desarrollo puberal. Los resultados se expresaron como valores de media y mediana e intervalo de confianza (IC del 95\%). En todos los casos se estableció un nivel de significación estadística en valores de $\mathrm{p}<0,05$.

El trabajo se llevó a cabo siguiéndose los principios de la Declaración de Helsinki y enmiendas relativas a la investigación en humanos. Se solicitó de cada participante la firma de un consentimiento informado por los padres o representantes legales. El estudio ha recibido el informe favorable del Comité Ético de Investigación Clínica del hospital donde se desarrolló.

\section{Resultados}

Teniendo en cuenta la población de referencia y los criterios de inclusión y exclusión se recogieron 39 pacientes durante el periodo establecido, de los que 3 no aceptaron participar o no completaron el protocolo del estudio, con lo que la muestra final fue de 36 pacientes. Respecto a las causas de movilidad reducida en nuestra muestra, el grupo más frecuente $(41,6 \%)$ fue secundario a encefalopatías de diversas etiologías (asociadas a cromosomopatías y síndromes malformativos, metabólicas e idiopáticas), seguido de la parálisis cerebral infantil $(33,3 \%)$, las enfermedades neuromusculares $(13,8 \%)$ y por último las de origen vascular $(11,1 \%)$.

Los principales resultados descriptivos de la muestra estudiada se recogen en la tabla 1. Destaca un valor medio disminuido en la densidad mineral ósea respec- to a los valores de referencia para edad y sexo. De este modo, el 47,2\% de la población estudiada presentó un $Z$ score inferior a -2, y sólo en el 33,3\% de los pacientes se obtuvo un $Z$ score superior a -1 , y de ellos en menos de la mitad (el 13,8\% del total de la muestra) el $\mathrm{Z}$ score fue superior a 0 . Aun así, ningún paciente de la muestra había presentado fracturas óseas previas. Se evidenciaron valores medios bajos en los parámetros de neoformación ósea, como la fosfatasa alcalina total y la osteocalcina, y niveles aumentados en los parámetros de reabsorción ósea como el NTX-I. En el resto de determinaciones, se observó que un 30,5\% de los pacientes de la muestra presentaba valores de 25-hidroxivitamina D3 $<20 \mathrm{ng} / \mathrm{ml}$ y el 16,6\% valores $<12 \mathrm{ng} / \mathrm{ml}$.

En la tabla 2 se representa la distribución de la puntuación del FMS en el grupo de estudio. Cabe destacar la afectación motora importante en buena parte de la muestra, de modo que el $33,3 \%$, el $44,3 \%$ y el $58,3 \%$ de los pacientes presentaron una puntuación de cero en las distancias de 5, 50 y 500 metros respectivamente.

Respecto a la relación entre la capacidad funcional y la densidad mineral ósea (tabla 3), se observó una asociación estadísticamente significativa entre la puntuación del FMS a los 5 metros y la densidad mineral ósea $(r=0,395 ; \mathrm{p}=0,017)$, así como entre la puntuación total del FMS y la densidad mineral ósea $(\mathrm{r}=0,365 ; \mathrm{p}=0,029)$. Por su parte, no se evidenció una correlación significativa entre los parámetros de neoformación - reabsorción ósea con la capacidad funcional (tabla 4) ni con la densidad mineral ósea (tabla 5). Tampoco se encontró relación entre el IMC y los valores de densidad mineral ósea $(r=0,268 ; p=0,115)$.

Por último, tampoco se observaron diferencias significativas al estratificar los resultados entre aquellos pacientes sin inicio puberal (estadio de Tanner 1) y aquellos otros con algún grado de desarrollo (estadios de Tanner 2 en adelante).

Tabla 1. Análisis descriptivo de la muestra analizada

\begin{tabular}{ll}
\hline Variables & Resultados \\
\hline Edad (años) (media, DE) & $8,6 \pm 4,7$ \\
Sexo (\%) & $52,7 \%$ mujeres, 47,3\% varones \\
Desarrollo puberal (estadios Tanner) (\%) & $1(67 \%), 2(11 \%), 3(17 \%), 4(5 \%)$ \\
IMC (Z score) (media, DE) & $-0,5 \pm 1,8$ \\
Densidad mineral ósea (Z score) (media, DE) & $-1,99 \pm 1,7$ \\
Fosfatasa alcalina total (Z score) (media, DE) & $-2,64 \pm 1,08$ \\
PTH (pg/ml) (media, DE) & $38,4 \pm 21,05$ \\
Osteocalcina (Z score) (media, DE) & $-2,15 \pm 1,39$ \\
NTX-I (Z score) (media, DE) & $3 \pm 1,72$ \\
25-hidroxi-vitamina D3 (ng/ml) (media DE) & $28,97 \pm 13,38$ \\
\hline
\end{tabular}

DE: Desviaciones estándar. IMC: Índice de masa corporal. PTH: Paratohormona. NTX-I: Telopéptido amino-terminal de cadena cruzada del colágeno tipo I. 
Tabla 2. Distribución de la puntuación del Functional Mobility Scale (FMS) en la muestra

\begin{tabular}{cccc}
\hline Puntuación & FMS 5 metros & FMS 50 metros & FMS 500 metros \\
\hline 0 & $12(33,3 \%)$ & $16(44,3 \%)$ & $21(58,3 \%)$ \\
1 & $7(19,4 \%)$ & $6(16,6 \%)$ & $6(16,6 \%)$ \\
2 & $5(13,8 \%)$ & $1(2,7 \%)$ & $12,7 \%)$ \\
3 & 0 & 0 & 0 \\
4 & $2(5,5 \%)$ & $4(11,1 \%)$ & $3(8,3 \%)$ \\
5 & $8(22,2 \%)$ & $8(22,2 \%)$ & $5(13,8 \%)$ \\
6 & $2(5,5 \%)$ & $1(2,7 \%)$ & 0 \\
\hline
\end{tabular}

Tabla 3. Relación entre capacidad funcional (FMS) y densidad mineral ósea (DMO)

\begin{tabular}{lcc}
\hline & Coeficiente correlación ${ }^{*}$ & p valor \\
\hline FMS 5 metros - DMO & 0,395 & 0,017 \\
FMS 50 metros - DMO & 0,385 & 0,071 \\
FMS 500 metros - DMO & 0,116 & 0,501 \\
FMS total - DMO & 0,365 & 0,029 \\
\hline
\end{tabular}

*Todos los análisis se realizaron empleando la correlación de Spearman.

Tabla 4. Relación entre capacidad funcional (FMS) y parámetros del metabolismo fosfocálcico analizados

\begin{tabular}{|c|c|c|c|c|c|c|c|c|}
\hline & \multicolumn{2}{|c|}{ FMS 5 metros } & \multicolumn{2}{|c|}{ FMS 50 metros } & \multicolumn{2}{|c|}{ FMS 500 metros } & \multicolumn{2}{|c|}{ FMS total } \\
\hline & $\begin{array}{l}\text { Coeficiente } \\
\text { correlación* }\end{array}$ & $p$ valor & $\begin{array}{l}\text { Coeficiente } \\
\text { correlación* }\end{array}$ & $p$ valor & $\begin{array}{l}\text { Coeficiente } \\
\text { correlación* }\end{array}$ & $\mathrm{p}$ valor & $\begin{array}{l}\text { Coeficiente } \\
\text { correlación* }\end{array}$ & $p$ valor \\
\hline Fosfatasa alcalina total & 0,209 & 0,222 & 0,127 & 0,461 & 0,132 & 0,443 & 0,174 & 0,310 \\
\hline PTH & 0,343 & 0,401 & 0,357 & 0,33 & 0,334 & 0,46 & 0,302 & 0,415 \\
\hline Osteocalcina & 0,201 & 0,239 & 0,083 & 0,630 & 0,136 & 0,428 & 0,179 & 0,297 \\
\hline NTX-I & $-0,231$ & 0,175 & -0.087 & 0,616 & 0,080 & 0,641 & $-0,177$ & 0,302 \\
\hline 25-OH- D3 & $-0,151$ & 0,379 & $-0,044$ & 0,800 & 0,018 & 0,915 & $-0,138$ & 0,422 \\
\hline
\end{tabular}

PTH: Paratohormona. NTX-I: Telopéptido amino-terminal de cadena cruzada del colágeno tipo I. 25-OH-D3: 25-hidroxi-vitamina D3. *Todos los análisis se realizaron empleando la correlación de Spearman.

\section{Discusión}

Teniendo en cuenta el predominio de la reabsorción ósea en las situaciones de movilidad reducida, se ha recogido un aumento del riesgo de fractura de un $4 \%$ por año en pacientes con parálisis cerebral infantil ${ }^{16}$, así como una prevalencia de fracturas de un 11 $30 \%$ en pacientes con espina bífida ${ }^{17}$ y de un $20,9-43 \%$ en pacientes con enfermedad de Duchenne ${ }^{18}$. En la muestra analizada, aunque no existían antecedentes de fracturas patológicas, sí se observó una densidad mineral ósea disminuida respecto a los valores de referencia para edad y sexo, así como una relación significativa entre la densidad mineral ósea y la capacidad funcional, sobre todo en el ámbito domiciliario (FMS a los 5 metros) y en la valoración global (FMS total).
Tabla 5. Relación entre densidad mineral ósea y parámetros del metabolismo fosfocálcico analizados

\begin{tabular}{lcc}
\hline & \multicolumn{2}{c}{ Densidad mineral ósea } \\
\cline { 2 - 3 } & $\begin{array}{c}\text { Coeficiente } \\
\text { correlación* }\end{array}$ & p valor \\
\hline Fosfatasa alcalina total & 0,029 & 0,868 \\
PTH & 0,215 & 0,203 \\
Osteocalcina & 0,313 & 0,063 \\
NTX-I & $-0,123$ & 0,474 \\
25-hidroxi-vitamina D3 & $-0,201$ & 0,239 \\
\hline
\end{tabular}

PTH: Paratohormona. NTX-I: Telopéptido amino-terminal de cadena cruzada del colágeno tipo I. *Todos los análisis se realizaron empleando la correlación de Pearson. 
A pesar de los beneficios de la actividad física sobre la masa ósea, no existe evidencia en la actualidad sobre los efectos del ejercicio en pacientes con movilidad reducida ${ }^{1,19}$. Aun así, se ha constatado un aumento de la densidad mineral ósea en pacientes con parálisis cerebral infantil tras prolongar el tiempo en bipedestación ${ }^{20}$, así como tras la realización de ejercicios de vibración de cuerpo entero ${ }^{21,22}$. Podrían tratarse de intervenciones prometedoras que pudieran emplearse como medidas preventivas o como complemento de otros tratamientos, aunque son necesarios estudios con mayor número de pacientes y más tiempo de seguimiento ${ }^{1}$.

En relación con el aumento de la actividad osteoclástica en los pacientes con movilidad reducida, en nuestro grupo de estudio se evidenció un incremento del NTX-I respecto a los valores de referencia, así como una disminución en parámetros de neoformación ósea como la osteocalcina o la fosfatasa alcalina total. Se considera que estos indicadores son predictores de la pérdida de masa ósea y del riesgo de presentar fracturas $^{23}$. De este modo, en pacientes adultos se ha observado una relación entre la osteocalcina, la fosfatasa alcalina y el NTX-I con la densidad mineral ósea ${ }^{24,25}$, hecho que no se ha constatado en nuestro trabajo. En uno de los pocos trabajos realizados en pacientes pediátricos, no se encuentra relación entre la osteocalcina y el estado funcional de un grupo de niños diagnosticados de mielomeningocele ${ }^{26}$, lo que coincide con los resultados de nuestro estudio. No obstante, conviene reseñar que estos marcadores de neoformación-reabsorción ósea son de difícil interpretación, ya que muestran una gran variabilidad dependiente de varios factores (edad, sexo e índice de masa corporal $)^{27}$. Por esta razón, su determinación sólo debe solicitarse en aquellos pacientes con riesgo elevado de presentar densidad mineral ósea disminuida y sus valores deben ajustarse a estos factores anteriormente $\operatorname{citados}^{28}$.

En cuanto a los niveles de vitamina $\mathrm{D}$, en nuestro grupo de estudio se observó que el 30,5\% de los pacientes presentaba un valor de 25-hidroxi-vitamina D3 < $20 \mathrm{ng} / \mathrm{ml}$, porcentaje que está dentro del rango de otros estudios realizados en nuestro país en población pediátrica, y que oscilan entre un $8,3 \%^{28}$ y un $52,7 \%{ }^{29}$. En ninguno de estos pacientes con insuficiencia o déficit de vitamina D se observó un hiperparatiroidismo secundario. Respecto a la influencia que la vitamina $\mathrm{D}$ tiene sobre la mineralización ósea en pacientes con enfermedades neuromusculares, se ha recogido una correlación positiva entre los valores de vitamina $\mathrm{D}$ y la densidad mineral ósea a nivel cortical ${ }^{1}$. Es necesario resaltar que estos pacientes con enfermedades neurológicas y alteración de la capacidad funcional constituyen un grupo de riesgo elevado para presentar insuficiencia de vitamina D debido a su mayor dificultad para salir al exterior $y$ conseguir una adecuada exposición a la luz solar, así como por los problemas nutricionales que presentan con frecuencia y al consumo de fármacos que pueden interferir en el metabolismo de la vitamina $\mathrm{D}$, como los corticoides o los anticonvulsivantes ${ }^{7}$. Por esta razón se ha establecido la recomendación de determinar los niveles de 25-hidroxi-vitamina D3 en aquellos casos con riesgo de presentar densidad mineral ósea disminuida, como es la inmovilización prolongada ${ }^{30}$.

Por tanto, se considera la movilidad reducida como una situación de riesgo para presentar densidad mineral ósea reducida y aumento del riesgo de fractura ${ }^{1,2,7,31}$. En este grupo de pacientes se recomienda que la densitometría ósea se realice cuando puedan beneficiarse de una intervención terapéutica y cuando los resultados puedan modificar la actitud clínica ${ }^{31}$. No obstante, las decisiones deben individualizarse, ya que la fragilidad ósea está influenciada por otros factores como la genética, la edad, el sexo o determinados fármacos ${ }^{7,31}$.

Como limitaciones del presente estudio, sería necesario comprobar nuestros resultados sobre la masa ósea en un mayor número de pacientes, así como ampliarlos a otros parámetros de neoformación-reabsorción ósea que no ha sido posible determinar en este trabajo, como la fosfatasa alcalina ósea, el propéptido amino-terminal del procolágeno tipo I, el telopéptido C-terminal del colágeno tipo I y la piridinolina. Con las nuevas técnicas de densitometría ósea podrían evidenciarse los efectos que la falta de movilidad produce por separado en el hueso cortical y en el trabecular.

Como conclusiones de nuestra serie de pacientes, cabe resaltar que se ha observado una disminución de la densidad mineral ósea dependiente del grado de movilidad, así como un predominio de los marcadores de reabsorción sobre los de neoformación ósea. Teniendo en cuenta que la adquisición de la masa ósea se lleva a cabo durante las dos primeras décadas de la vida, podrían desarrollarse estrategias encaminadas a realizar un diagnóstico precoz de los problemas de salud ósea en estos pacientes con movilidad reducida para así prevenir complicaciones en los años posteriores.

\section{Responsabilidades Éticas}

Protección de personas y animales: Los autores declaran que los procedimientos seguidos se conformaron a las normas éticas del comité de experimentación humana responsable y de acuerdo con la Asociación Médica Mundial y la Declaración de Helsinki.

Confidencialidad de los datos: Los autores declaran que han seguido los protocolos de su centro de trabajo sobre la publicación de datos de pacientes.

Derecho a la privacidad y consentimiento informado: Los autores han obtenido el consentimiento in- 
formado de los pacientes y/o sujetos referidos en el artículo. Este documento obra en poder del autor de correspondencia.

\section{Conflicto de intereses}

Los autores declaran no tener conflicto de intereses.

\section{Referencias}

1. Yasar E, Adigüzel E, Arslan M, Matthews DJ. Basics of bone metabolism and osteoporosis in common pediatric neuromuscular disabilities. Eur J Paediatr Neurol. 2018;22(1):17-26.

2. Saraff V, Högler W. Osteoporosis in children: diagnosis and management. Eur J Endocrinol. 2015;173(6):185-97.

3. Frost HM. Bone`s mechanostat: a 2003 update. Anat Rec A Discov Mol Cel Evol Biol. 2003;275:1081-1101.

4. Frost HM, Schönau E. The "Muscle-Bone Unit” in children and adolescents: a 2000 overview. J Pediatr Endocrinol Metab. 2000;13:571-90.

5. Zacharin M. Current advances in bone health of disabled children. Curr Opin Pediatr. 2004;16:545-51.

6. Binckley T, Johnson J, Vogel L, Kecskemethy H, Henderson R, Specker B. Bone measurements by peripheral quantitative computer tomography (pQCT) in children with cerebral palsy. J Pediatr. 2005;147:791-6.

7. Ness K, Apkon SD. Bone health in children with neuromuscular disorders. J Pediatr Rehabil Med. 2014;7(2):133-42.

8. Golden NH, Abrams SA; Committee on Nutrition. Optimizing bone health in children and adolescents. Pediatrics. 2014;134(4):e1229-43.

9. Graham HK, Harvey A, Rodda J, Nattrass GR, Pirpiris M. The Functional Mobility Scale (FMS). J Pediatr Orthop. 2004;24(5):514-20.

10. Carrascosa A, Yeste D, Moreno-Galdó A, et al. Índice de masa corporal e índice de masa triponderal de 1453 niños no obesos ni malnutridos de la generación del milenio. Estudio longitudinal de Barcelona. An Pediatr (Barc). 2018;89(3):137-43.

11. Del Río L, Carrascosa A, Pons F, Gussinye M, Yeste D, Domenec FM. Bone mineral density in lumbar spine in caucasian mediaterraean spanish children and adolescents. Changes related to age, sex and puberty. Pediatr Res. 1994;35:362-6.

12. Rauch F, Stabrey A, Schönau E. Appendix: Reference values in pediatric osteology. En: Pediatric Osteology: New developments in diagnostics and therapy. Schönau E (ed). Amsterdam, Elsevier Science BV, 1996:295-300.

13. Cortés Blanco A, Labarta Aizpún JI, Ferrández Longás A, Mayayo Dehesa E. Reference values for IGF-I, IGFBP-1, IGFBP-3 and osteocalcin in healthy children in Zaragoza. An Esp Pediatr. 1999;51(2):167-74.

14. Bollen AM, Eyre DR. Bone resorption rates in children monitored by the urinary assay of collagen type I cross-linked peptides. Bone. 1994;15(1):31-4

15. Munns CF, Shaw N, Kiely M, et al. Global Consensus Recommendations on Prevention and Management of Nutritional Rickets. J Clin Endocrinol Metab. 2016;101(2):394-415.

16. Stevenson RD, Conaway M, Barrington JW, Cuthill SL, Worley G, Henderson RC. Fracture rate in children with cerebral palsy. Pediatr Rehabil. 2006;9(4):396-403.

17. Szalay EA, Cheema A. Children with spina bifida are at risk for low bone density. Clin Orthop Relat Res. 2011;469(5):12537.

18. McDonald DG, Kinali M, Gallagher AC, et al. Fracture prevalence in Duchenne muscular dystrophy. Dev Med Child Neurol. 2002;44(10):695-8.

19. Ozel S, Switzer L, Macintosh A, Fehlings D. Informing evidence-based clinical practice guidelines for children with cerebral palsy at risk of osteoporosis: an update. Dev Med Child Neurol. 2016;58(9):918-23.

20. Caulton JM, Ward KA, Alsop CW, Dunn G, Adams JE, Mughal MZ. A randomised controlled trial of standing programme on bone mineral density in non-ambulant children with cerebral palsy. Arch Dis Child. 2004;89(2):131-5.

21. Wren TA, Lee DC, Hara R, et al. Effect of high-frequency, low-magnitude vibration on bone and muscle in children with cerebral palsy. J Pediatr Orthop. 2010;30(7):732-8.

22. Reyes ML, Hernández M, Holmgren LJ, Sanhueza E, Escobar RG. High-frequency, low-intensity vibrations increase bone mass and muscle strength in upper limbs, improving autonomy in disabled children. J Bone Miner Res. 2011;26(8):1759-66.

23. Vasikaran $\mathrm{S}$, Eastell $\mathrm{R}$, Bruyère $\mathrm{O}$, et al. Markers of bone turnover for the prediction of fracture risk and monitoring of osteoporosis treatment: a need for international reference standards. Osteoporos Int. 2011;22(2):391-420.

24. Atalay S, Elci A, Kayadibi H, Onder CB, Aka N. Diagnostic utility of osteocalcin, undercarboxylated osteocalcin, and alkaline phosphatase for osteoporosis in premenopausal and postmenopausal women. Ann Lab Med. 2012;32(1): 23-30.

25. Baxter I, Rogers A, Eastell R, Peel N. Evaluation of urinary $\mathrm{N}$-telopeptide of type I collagen measurements in the management of osteoporosis in clinical practice. Osteoporos Int. 2013;24(3):9417.

26. Okurowska-Zawada B, Kozerska A, Żelazowska B, Kułak W, Wasilewska A, Wysocka J. Serum 25-hydroxyvitamin $\mathrm{D}$, osteocalcin, and parathormone status in children with meningomyelocele. Neuropediatrics. 2012;43(6):314-9.

27. Hlaing TT, Compston JE. Biochemical markers of bone turnover - uses and limitations. Ann Clin Biochem 2014; 51:189-202.

28. Togo A, Espadas Maciá D, Blanes Segura S, Sivó Díaz N, Villalba Martínez C. Is there vitamin $\mathrm{D}$ deficiency in children in a sunny Mediterranean city?. An Pediatr (Barc). 2016;84(3):163-9.

29. Rodriguez-Dehli AC, Riaño-Galán I, Fernández-Somoano A, et al. Hipovitaminosis D and associated factors in 4 year-old children in northern Spain. An Pediatr (Barc). 2017;86(4):188-96.

30. Harel Z, Cromer B, DiVasta AD, Gordon CM. Recommended vitamin D intake and management of low vitamin D status in adolescents: a position statement of the society for adolescent health and medicine. J Adolesc Health. 2013;52(6):801-3.

31. Bachrach LK, Gordon CM. Bone Densitometry in Children and Adolescents. Pediatrics. 2016;138(4). pii: e20162398. 